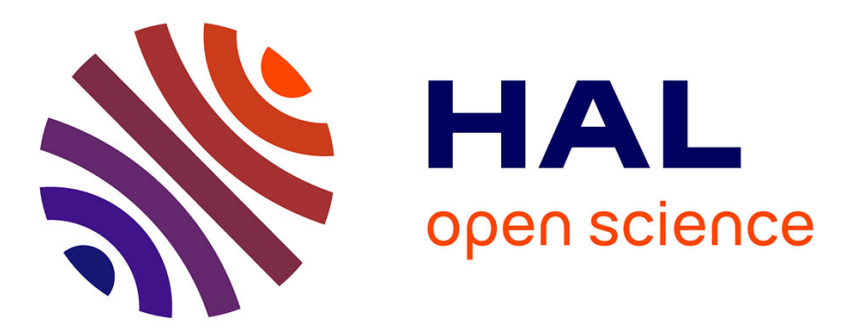

\title{
Wind estimation algorithm for quadrotors using detailed aerodynamic coefficients
}

\author{
Gabriele Perozzi, Denis Efimov, Jean-Marc Biannic, Laurent Planckaert, \\ Patricia Coton
}

\section{- To cite this version:}

Gabriele Perozzi, Denis Efimov, Jean-Marc Biannic, Laurent Planckaert, Patricia Coton. Wind estimation algorithm for quadrotors using detailed aerodynamic coefficients. ACC 2018 - American Control Conference, Jun 2018, Milwaukee, WI, United States. hal-01703084

\section{HAL Id: hal-01703084 \\ https://hal.inria.fr/hal-01703084}

Submitted on 7 Feb 2018

HAL is a multi-disciplinary open access archive for the deposit and dissemination of scientific research documents, whether they are published or not. The documents may come from teaching and research institutions in France or abroad, or from public or private research centers.
L'archive ouverte pluridisciplinaire HAL, est destinée au dépôt et à la diffusion de documents scientifiques de niveau recherche, publiés ou non, émanant des établissements d'enseignement et de recherche français ou étrangers, des laboratoires publics ou privés. 


\title{
Wind estimation algorithm for quadrotors using detailed aerodynamic coefficients
}

\author{
Gabriele Perozzi, Denis Efimov, Jean-Marc Biannic, Laurent Planckaert, Patricia Coton
}

\begin{abstract}
In the context of safe control of quadrotors, wind velocity estimation and compensation have a key-role. For this reason, assuming the lack of airspeed sensors and considering sensors noise, in this paper three time-varying parameter estimation algorithms are introduced, studied and merged to estimate the varying wind velocity, using only on-board quadrotor sensors and an inertial tracking position system (e.g. Optitrack camera, GPS). To this end, a detailed quadrotor flight dynamics model is presented using identified aerodynamic coefficients and wind velocity components along the three axes. Then, a decomposition of dynamical equations is performed in known and unknown terms to be estimated. Thanks to this decomposition, the estimation algorithms are built and finally tested and validated in numerical experiments, against the introduced sensors' noise.
\end{abstract}

\section{INTRODUCTION}

Quadrotors are usually required to move in environments where wind velocity is unknown. To improve their stability, a good control must ensure a good robustness against the disturbance caused by this wind. The problem is that, nowadays the pressure sensors, such as aeroclinometer, Pitot tube and LIDAR based sensors, are too heavy to be mounted on a mini drone. For this reason, an estimation software scheme (or an intelligent sensor) has to be designed based on measurements available on quadrotors and on inertial tracking position system. Then, the objective of this work is to develop an on-board algorithm for estimation of time-varying wind parameters by taking into account a detailed physical model. The design objectives include the time convergence optimization and robustness to measurement noises and model uncertainties improvement. The estimation is performed using IMU (accelerometer, gyroscope) sensors augmented with ground based camera system and rotors angular velocities sensors.

According to the aerodynamic science, a nonlinear dependence of the UAV by the wind speed comes out, while the disturbances (external forces and moments) enter linearly in the drone equations. To estimate constant and time-varying parameters, many algorithms were proposed recently in the literature, and two main groups can be identified:

The following works estimate the wind velocity using airspeed sensors. In [3], the high-order sliding mode observer

G. Perozzi, L. Planckaert, P. Coton are with ONERA - The French Aerospace Lab, DAAA, 5 Bd Paul Painlevé BP 2126159014 Lille Cedex, France.

D. Efimov is with Inria, Non-A team, Parc Scientifique de la Haute Borne, 40 avenue Halley, Bât. A, Park Plaza, 59650 Villeneuve d'Ascq, France.

JM. Biannic is with ONERA - The French Aerospace Lab, DTIS, BP74025 2 avenue Edouard Belin 31055 Toulouse Cedex 4, France.

This work was funded by Hauts-de-France region and ONERA. is constructed as an estimator of the effect of the external disturbances in quadrotors such as wind and noise. Another algorithm is provided by [4] which develops an extension of the Kalman filter that provides an estimate of the unknown wind disturbance in micro-UAV scenario. The paper [5] estimates the wind velocity, angle-of-attack and sideslip angle of a fixed-wing UAV using kinematic relationships with a Kalman Filter, avoiding the need to know aerodynamic models or other aircraft parameters. The paper [6] describes a method for estimating wind velocity, rate of change of wind velocity, and wind gradient for small and mini UAVs. In the work [8] an observer estimates wind data, which is used in the case where the UAV subjected to wind perturbation has to follow an object on the ground.

The following articles propose wind and disturbance estimations without the use of additional airspeed sensors. The paper [1] presents Lyapunov method for external forces and moments in flying robots. The article [2] presents a modelbased method for external wrench estimation in flying robots based on proprioceptive sensors and the robot's dynamics model. The article [7] develops an estimation algorithm for the wind velocity using the inversion of the equations that define the components of the accelerometer measurements.

The paper outline is as follows. In Section II the considered UAV drone is described and the flight dynamics model is derived. A preliminary study of the dynamics is carried out in Section III. The wind estimation algorithms are presented in Section IV. The results of numeric experiments are shown in Section V. The remarks and discussion conclude the paper in Section VI.

\section{FLIGHT DYNAMICS}

This section presents the model of the UAV dynamics, which has configuration as it is shown in Fig. 1.

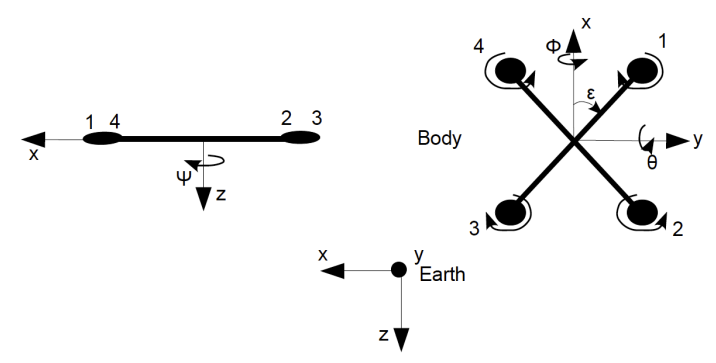

Fig. 1. UAV configuration

According to the identification work at low/medium velocity in [9] using the Parrot AR Drone 2.0 and similar, rotors 
gyroscopic effects and inertial counter torques are neglected since they are rather small. The translational dynamics of the drone in the body frame yield

$$
m \dot{\mathbf{u}}+m \varpi \times \mathbf{u}=\mathbf{F}_{\text {aero }}+m \mathbf{R}^{T} \mathbf{g},
$$

where $m$ is the mass of the $\mathrm{UAV}, \mathbf{u}=[u v w]^{T}$ is its linear velocity expressed in body frame, $\varpi=\left[\begin{array}{ll}p & q\end{array}\right]^{T}$ is its angular velocity in body frame, $\mathbf{F}_{\text {aero }}=\left[F_{\text {Xaero }} F_{\text {Yaero }} F_{\text {Zaero }}\right]^{T}$ is the vector of the external aerodynamic forces in body frame, $\mathbf{g}=\left[\begin{array}{lll}0 & 0 & g\end{array}\right]^{T}$ is the gravity acceleration in inertial frame, $\mathbf{R}$ is the rotational matrix defining the passage between the inertial and body frames. The rotational dynamics of the drone with respect to inertial earth frame are

$$
\mathbf{I} \dot{\varpi}=-\varpi \times \mathbf{I} \varpi+\tau_{\text {aero }}
$$

where $\mathbf{I}$ is the inertia matrix of the UAV, and $\tau_{\text {aero }}=$ $\left[L_{\text {aero }} M_{\text {aero }} N_{\text {aero }}\right]^{T}$ is the external aerodynamic moments in the body frame. The relation between angular velocities and Euler angles

$$
\begin{gathered}
\dot{\phi}=p+\tan \theta(q \sin \phi+r \cos \phi), \\
\dot{\theta}=q \cos \phi-r \sin \phi, \\
\dot{\psi}=\frac{q \sin \phi+r \cos \phi}{\cos \theta},
\end{gathered}
$$

are considered avoiding the singularities $\theta \neq \frac{\pi}{2}$, which is a reasonable assumption in our case since the topic of this article is not to achieve aggressive maneuvers. The full model of the system is presented by the equations (1), (2), (3).

The aerodynamic forces, moments, and coefficients are derived using a combination of momentum and blade element theory in helicopters, explained by [10], [11], [12]. Aerodynamic forces and moments for each rotor, where subscript $j$ indicates the $j^{\text {th }}$ rotor, are derived as

$$
\begin{aligned}
F_{X j} & =-\rho A R^{2} \frac{u_{j}-u_{w}}{\sqrt{\left(u_{j}-u_{w}\right)^{2}+\left(v_{j}-v_{w}\right)^{2}}} C_{H j} \omega_{j}^{2}, \\
F_{Y j} & =-\rho A R^{2} \frac{v_{j}-v_{w}}{\sqrt{\left(u_{j}-u_{w}\right)^{2}+\left(v_{j}-v_{w}\right)^{2}}} C_{H j} \omega_{j}^{2}, \\
F_{Z_{j}} & =-\rho A R^{2} C_{T j} \omega_{j}^{2} \\
L_{j} & =-\operatorname{sign} \omega_{j} \rho A R^{3} \frac{u_{j}-u_{w}}{\sqrt{\left(u_{j}-u_{w}\right)^{2}+\left(v_{j}-v_{w}\right)^{2}}} C_{R m_{j}} \omega_{j}^{2}, \\
M_{j} & =-\operatorname{sign} \omega_{j} \rho A R^{3} \frac{v_{j}-v_{w}}{\sqrt{\left(u_{j}-u_{w}\right)^{2}+\left(v_{j}-v_{w}\right)^{2}}} C_{R m_{j}} \omega_{j}^{2}, \\
N_{j} & =-\operatorname{sign} \omega_{j} \rho A R^{3} C_{Q_{j}} \omega_{j}^{2},
\end{aligned}
$$

where $\rho$ is the air density, $A$ is the rotor area, $R$ is the rotor radius, $\left[u_{w} v_{w} w_{w}\right]$ is the wind velocity with respect to the earth in body frame, $C_{H}$ is the hub force coefficient, $C_{T}$ is rotor thrust coefficient, $\omega$ is the rotor angular speed, $C_{Q}$ is the rotor drag moment coefficient, $C_{R m}$ is the rotor rolling moment coefficient. Total aerodynamic forces are

$$
F_{X \text { aero }}=\sum_{j=1}^{4} F_{X j}, \quad F_{\text {Yaero }}=\sum_{j=1}^{4} F_{Y j}, \quad F_{\text {Zaero }}=\sum_{j=1}^{4} F_{Z_{j}}
$$

Total aerodynamic moments are

$$
\begin{gathered}
L_{\text {aero }}=\sum_{j=1}^{4}\left(L_{j}+F_{Z_{j}} l \mathrm{~s}_{j}-h F_{Y j}\right), \\
M_{\text {aero }}=\sum_{j=1}^{4}\left(M_{j}-F_{Z_{j}} l \mathrm{c}_{j}+h F_{X_{j}}\right) \\
N_{\text {aero }}=\sum_{j=1}^{4}\left(N_{j}+F_{Y_{j}} l \mathrm{c}_{j}-F_{X j} l \mathrm{~s}_{j}\right),
\end{gathered}
$$

where $h$ is the distance between rotors plane and the center of gravity of the UAV, $l$ is the arm length, and with $\mathrm{c}_{j}=\cos \left(\frac{\pi}{2}(j-1)+\varepsilon\right), \mathrm{s}_{j}=\sin \left(\frac{\pi}{2}(j-1)+\varepsilon\right)$. In our UAV configuration we have $\varepsilon=\frac{\pi}{4}$, thus for vectors $\mathrm{c}_{j}$ and $\mathrm{s}_{j}$ we have cosines and sinus of the angles $\left[\frac{\pi}{4}, \frac{3}{4} \pi, \frac{5}{4} \pi, \frac{7}{4} \pi\right]$. According to [9] thanks to in-door experiments on Parrot drone, in moderate velocity and in climbing phase, the aerodynamic coefficients are identified as follows

$$
\begin{gathered}
C_{T j}=C_{T_{s t a t}}+K_{z} \frac{w_{j}-w_{w}}{R\left|\omega_{j}\right|}, \quad C_{T_{\text {stat }}}>0, \\
\lambda_{j}=\lambda_{\text {stat }}-\frac{4}{\sigma a} K_{z} \frac{w_{j}-w_{w}}{R\left|\omega_{j}\right|}, \quad K_{z} \geq 0, \lambda_{\text {stat }}>0, \\
\mu_{j}=\frac{1}{\left|\omega_{j}\right| R} \sqrt{\left(u_{j}-u_{w}\right)^{2}+\left(v_{j}-v_{w}\right)^{2}}, \\
C_{R m j}=\sigma a \frac{\mu_{j}}{8}\left(\lambda_{j}-\frac{4}{3}\right), \\
\frac{\sigma C_{D 0}}{8}\left(1+\mu_{j}^{2}\right)+\sigma a \lambda_{j}\left(\frac{\theta_{0}}{6}-\frac{\lambda_{j}}{4}\right), \\
C_{H j}=K_{D} \mu_{j}, \quad K_{D} \geq 0
\end{gathered}
$$

where $\sigma$ is the rotor solidity ratio, $a$ is the lift curve slope of the blade section, $C_{D 0}$ is the drag coefficient of the blade section, $\theta_{0}$ is the angle of attack of the root profile, $\lambda$ is the inflow ratio, $\mu$ is the advance ratio, subscript stat indicates the value in hover phase. The above UAV coefficients can be explained recalling aerodynamic science.

\section{PRELIMINARIES}

Substituting (6), (4) and (5) in (1), (2), the UAV dynamics equations can be rewritten in state-space form

$$
\dot{X}=f\left(X, U, \omega, d_{w}\right),
$$

where $f$ is expressed in (1), (2), $d_{w}=\left[\begin{array}{lll}u_{w} & v_{w} & w_{w}\end{array}\right]^{T}$ is the vector of time-varying wind velocities to be estimated, $\omega$ is the vector of rotors angular rotational velocities and they are directly measured by sensors, the control vector $U$ contains the terms proportional to the squares of rotors velocities $\omega_{j}^{2}$, and the state $X=[u v w p q r]^{T}$ is represented by the linear and angular velocities of the UAV in the body frame. Explicit mathematical computations are illustrated in [13], [14]. Linear velocities $u, v, w$ together with their derivatives (accelerations) are provided by the on-board accelerometer, which measures directly

$$
\tilde{\Delta}_{a}(X)=\Delta_{a}(X)+\varepsilon_{a}
$$

$\Delta_{a}(X)=\left[\begin{array}{c}\Delta_{a u}(X) \\ \Delta_{a v}(X) \\ \Delta_{a w}(X)\end{array}\right]=\left[\begin{array}{c}\dot{u} \\ \dot{v} \\ \dot{w}\end{array}\right]+\left[\begin{array}{c}p \\ q \\ r\end{array}\right] \times\left[\begin{array}{c}u \\ v \\ w\end{array}\right]-\left[\begin{array}{c}-g \sin \theta \\ g \cos \theta \sin \phi \\ g \cos \theta \cos \phi\end{array}\right]$, 
where $\varepsilon_{a}$ is a bounded measurement noise of the accelerometer. From the gyroscope, which measures the rotational velocity in body frame with respect to the earth, the rest state coordinates are measured

$$
\begin{aligned}
\tilde{\Delta}_{g}(X) & =\Delta_{g}(X)+\varepsilon_{g}, \\
\Delta_{g}(X) & =\left[\begin{array}{lll}
p & q & r
\end{array}\right]^{T},
\end{aligned}
$$

where $\varepsilon_{g}$ is the measurement noise generated by gyroscope. IMU sensor is augmented with ground based cameras, used to estimate $u, v, w, \phi, \theta$ in coupling with gyroscope and accelerometer and making the drone observable with respect to the inertial frame.

Following the structure of measured information, another decomposition can be performed by splitting the dynamic equations in two parts

$$
\left[\begin{array}{c}
\Delta_{a}(X) \\
\dot{\Delta}_{g}(X)
\end{array}\right]=f_{0}(X, U, \omega)+\Omega(\omega) d_{w},
$$

where $f_{0}: \mathbb{R}^{14} \rightarrow \mathbb{R}^{6}$ is supposed to be known, and $\Omega: \mathbb{R}^{4} \rightarrow$ $\mathbb{R}^{6 \times 3}$ is a time-varying regressor matrix related to the wind speed $d_{w}$, which has to be estimated. In order to derive the expressions of $f_{0}$ and $\Omega$, the translational rotor velocities in the body frame are computed as

$$
\left[\begin{array}{c}
u_{j}(X) \\
v_{j}(X) \\
w_{j}(X)
\end{array}\right]=\left[\begin{array}{l}
p \\
q \\
r
\end{array}\right] \times\left[\begin{array}{c}
l c_{j} \\
l s_{j} \\
h
\end{array}\right]+\left[\begin{array}{c}
u \\
v \\
w
\end{array}\right] .
$$

Remark 1: Nonlinear terms, which represent a small part of rotor rolling torque $R_{m}$ for roll

$$
+\operatorname{sign}\left(\omega_{j}\right) \frac{1}{2} \rho A R K_{z}\left(u_{j}-u_{w}\right)\left(w_{j}-w_{w}\right)
$$

and for pitch

$$
+\operatorname{sign}\left(\omega_{j}\right) \frac{1}{2} \rho A R K_{z}\left(v_{j}-v_{w}\right)\left(w_{j}-w_{w}\right),
$$

and a small part of rotor torque $Q$ for yaw dynamics

$$
\begin{gathered}
-\operatorname{sign} \omega_{j} \rho A R \\
\times\left(\frac{1}{8} \sigma C_{D 0}\left(\left(u_{j}-u_{w}\right)^{2}+\left(v_{j}-v_{w}\right)^{2}\right)-\frac{4 K_{z}^{2}}{\sigma a}\left(w_{j}-w_{w}\right)^{2}\right)
\end{gathered}
$$

are neglected. These restrictions can be accepted for low UAV and wind velocities.

It is worth noting that the provided UAV model allows the wind estimation using only three translational dynamics with a simple inversion. Rotational dynamics adds three equations to improve the estimation, since inside the translational dynamics we already have terms proportional to $\theta, \phi$ that consider the rotational behavior of the UAV subjected to external wind. Moreover the rotational dynamics include also the inertia matrix which is rather complicated to precisely estimate.

\section{ESTIMATION ALGORITHM}

In the following, the argument dependence of the functions $f_{0}$ and $\Omega$ is avoided to make the presentation more compact. To design the algorithm let us decompose the system (7) as follows

$$
\begin{aligned}
& {\left[\begin{array}{c}
\Delta_{a} \\
\dot{\Delta}_{g}
\end{array}\right]=\left[\begin{array}{l}
f_{0 a} \\
f_{0 g}
\end{array}\right]+\left[\begin{array}{l}
\Omega_{a} \\
\Omega_{g}
\end{array}\right] d_{w},} \\
& {\left[\begin{array}{c}
\tilde{\Delta}_{a} \\
\tilde{\Delta}_{g}
\end{array}\right]=\left[\begin{array}{l}
\Delta_{a}+\varepsilon_{a} \\
\Delta_{g}+\varepsilon_{g}
\end{array}\right],}
\end{aligned}
$$

where the variables $\tilde{\Delta}_{a}$ and $\tilde{\Delta}_{g}$ are available from measurements, $f_{0 a}, \Omega_{a}, f_{0 g}, \Omega_{g}$ are the vector and matrix variables, respectively for translational (subscript $a$ ) and rotational (subscript $g$ ), whose values are functions of measured variables $X, U, \omega$. The presented algorithms are designed assuming that $u, v, w$, and $\phi, \theta$ with their derivatives are available from measurements (trajectography, optical flow, GPS). For simplicity, in theoretical analysis we supposed that these values are reconstructed exactly, but for simulation a state measurement noise has been added modeling this effect. To avoid unnecessary state differentiation, three sub-algorithms can be considered: one suitable for translational dynamics, the others for rotational dynamics. The algorithms presented in this section are based on the following hypothesis (we will switch between them depending on applied approach):

Assumption 1: The measurement noises are absent $\left(\varepsilon_{a}=0\right.$ and $\left.\varepsilon_{g}=0\right)$ and the wind velocity is constant $\left(\dot{d}_{w}=0\right)$.

Assumption 2: The measurement noises $\varepsilon_{a}, \varepsilon_{g}$ and the wind acceleration $\dot{d}_{w}$ are bounded signals

$$
\sup _{t \geq 0} \max \left\{\left|\varepsilon_{a}(t)\right|,\left|\varepsilon_{g}(t)\right|\right\} \leq \bar{\varepsilon}, \quad \sup _{t \geq 0}\left|\dot{d}_{w}(t)\right| \leq \bar{d}_{w},
$$

for some $\bar{\varepsilon}>0$ and $\bar{d}_{w}>0$.

Assumption 3: The matrix $\Omega_{g}$ is bounded and persistently excited for all $t \geq 0$ (see [15] for definition of this property ${ }^{1}$ ).

\section{A. Translational dynamics}

Define the predicted acceleration:

$$
\hat{\Delta}_{a}=f_{0 a}+\Omega_{a} \hat{d}_{w}
$$

which is based on the estimate $\hat{d}_{w}$ of the wind velocity derived below; and introduce the error between the measured state acceleration $\tilde{\Delta}_{a}$ and predicted one as follows

$$
e_{a}=\tilde{\Delta}_{a}-\hat{\Delta}_{a}=\Omega_{a}\left(d_{w}-\hat{d}_{w}\right)+\varepsilon_{a},
$$

with $\varepsilon_{a}$ the bounded sensors noise for the accelerometer. According to [16], the following finite-time estimation algorithm can be introduced

$$
\dot{\hat{d}}_{w}=\gamma_{a} \Omega_{a}^{T}\left\lceil e_{a}\right\rfloor^{\alpha}, \quad 0<\alpha<1, \gamma_{a} \gg 0,
$$

where $\lceil\cdot\rfloor^{\alpha}=|\cdot|^{\alpha} \operatorname{sign}(\cdot)$ is understood element-wise. The following results can be proven (the proofs are excluded due to space limitations).

\footnotetext{
${ }^{1}$ The Lebesgue measurable and square integrable matrix function $R$ is PE if there are $\ell>0, \xi>0$ such that $\int_{t}^{t+\ell} R(s) R(s)^{T} d s \geq \xi_{\ell}$, where $I_{\ell}$ is an identity square matrix of dimension $\ell$.
} 
Theorem 1: Let Assumption 1 be satisfied, then the value $d_{w}$ can be estimated in a finite time by (9).

Theorem 2: Let Assumption 2 be satisfied, then for (9) there exist $T\left(\bar{d}_{w}, \bar{\varepsilon}\right)>0$ and $v_{a 2}>0$ such that

$$
\left|d_{w}(t)-\hat{d}_{w}(t)\right| \leq v_{a 2} \max \left\{\bar{d}_{w}, \bar{\varepsilon}\right\} \quad \forall t \geq T\left(\bar{d}_{w}, \bar{\varepsilon}\right) .
$$

Therefore, the system (9) is globally finite-time stable, and the parameter identification error converges to a neighborhood of the origin that depends on the upper bound of the noise $\bar{\varepsilon}$, maximal amplitude of acceleration of the wind $\overline{\dot{d}}_{w}$, the choice of the gain $\gamma_{a}$ and the parameter $\alpha$.

\section{B. Rotational dynamics}

Wind estimation problem for rotational dynamics is slightly different from the linear one because the state vector is measured and not its derivative is provided by the sensor, hence an adaptive observer, which estimates the state and the wind simultaneously, has to be also designed. To this end, first, the adaptive observer equations can be written as follows

$$
\begin{aligned}
& \dot{\hat{\Delta}}_{g}=f_{0 g}+\Omega_{g} \hat{d}_{w}+\ell \operatorname{sign}\left(\tilde{\Delta}_{g}-\hat{\Delta}_{g}\right), \\
& \dot{\hat{d}}_{w}=\gamma_{g} \Omega_{g}^{T}\left(\tilde{\Delta}_{g}-\hat{\Delta}_{g}\right),
\end{aligned}
$$

where $\hat{\Delta}_{g}$ is an estimate of the state vector $\Delta_{g}, \hat{d}_{w}$ is again an estimate of the vector of wind velocities $d_{w} ; \ell>0$ and $\gamma_{g} \gg 0$ are tuning parameters. The following result can be obtained (the induced norm of a matrix $\Omega_{g}$ is denoted by $\left.\left\|\Omega_{g}\right\|_{2}\right)$ :

Theorem 3: Let assumptions 1 and 3 be satisfied, and there is a known bound $\bar{d}_{w}>0$ such that $\left|d_{w}\right| \leq \bar{d}_{w}$, then for $\ell>2\left\|\Omega_{g}\right\|_{2} \bar{d}_{w}$ the estimate $\hat{d}_{w}$ in (10) converges to the value of $d_{w}$ in a finite time.

The main issue with the algorithm (10) is hidden in rather strong restrictions imposed in Assumption 1, which we need to substantiate the convergence. Less restricted estimation algorithm can be obtained by the price of an augmented computational complexity as the following one:

$$
\begin{aligned}
\dot{\hat{\Delta}}_{g} & =f_{0 g}+\Omega_{g} \hat{d}_{w}+\ell^{\prime}\left(\tilde{\Delta}_{g}-\hat{\Delta}_{g}\right)+\Xi \dot{\hat{d}_{w}}, \\
\dot{\Xi} & =-\ell^{\prime} \Xi+\Omega_{g}, \\
\dot{\hat{d}}_{w} & =\gamma_{g}^{\prime} \Xi^{T}\left[\tilde{\Delta}_{g}-\hat{\Delta}_{g}\right\rfloor^{\alpha^{\prime}},
\end{aligned}
$$

where $\hat{\Delta}_{g}$ and $\hat{d}_{w}$ as before are the estimates of $\Delta_{g}$ and $d_{w}$, respectively; $\ell^{\prime}>0, \alpha^{\prime} \in(0,1)$ and $\gamma_{g}^{\prime} \gg 0$ are design parameters; $\Xi$ is an auxiliary matrix variable having the dimension of $\Omega_{g}$ (obviously it is always bounded for bounded $\Omega_{g}$ and $\left.\ell^{\prime}>0\right)$ and having its initialization in $\Xi(0) \neq 0$. In order to clarify the stability and robustness properties of this estimation scheme let us introduce three estimation errors

$$
e_{\Delta}=\Delta_{g}-\hat{\Delta}_{g}, \quad e_{d}=d_{w}-\hat{d}_{w}, \quad \delta=e_{\Delta}-\Xi e_{d},
$$

which have the following dynamics:

$$
\begin{aligned}
\dot{e}_{\Delta} & =-\ell^{\prime}\left(e_{\Delta}+\varepsilon_{g}\right)+\Omega_{g} e_{d}-\gamma_{g}^{\prime} \Xi \Xi^{T}\left\lceil e_{\Delta}+\varepsilon_{g}\right\rfloor^{\alpha^{\prime}}, \\
\dot{e}_{d} & =-\gamma_{g}^{\prime} \Xi^{T}\left\lceil\Xi e_{d}+\delta+\varepsilon_{g}\right\rfloor^{\alpha^{\prime}}+\dot{d}_{w}, \\
\dot{\delta} & =-\ell^{\prime}\left(\delta+\varepsilon_{g}\right)-\Xi \dot{d}_{w} .
\end{aligned}
$$

Introduce the following hypothesis:

Assumption 4: The minimum singular value of the matrix variable $\Xi(t) \in \mathbb{R}^{3 \times 3}$ is bigger than $\sigma_{\Xi}$ for all $t \geq 0$.

The last condition on $\Xi$ can be ensured by a proper initialization and the same property of $\Omega_{g}$ (Assumption 3). The following result can be substantiated:

Theorem 4: Let assumptions 2 and 4 be satisfied, then in (11) there exists $T>0$ such that

$$
\begin{aligned}
& \left|e_{d}(t)\right| \leq \rho\left(\bar{\varepsilon}, \bar{d}_{w}\right), \\
& \left|e_{\Delta}(t)\right| \leq \max \left\{\sigma_{\Xi}^{-1} \bar{\varepsilon}, \frac{\sqrt{2}}{\ell^{\prime}}\left(\left\|\Omega_{g}\right\|_{2} \rho\left(\bar{\varepsilon}, \bar{d}_{w}\right)+\ell^{\prime} \bar{\varepsilon}\right)\right\},
\end{aligned}
$$

for all $t \geq T$, where

$$
\begin{gathered}
\rho\left(\bar{\varepsilon}, \overline{\dot{d}}_{w}\right)=\sigma_{\Xi}^{-1} \max \left\{\frac{2}{\ell^{\prime}}\|\Xi\|_{2} \overline{\dot{d}}_{w}+3 \bar{\varepsilon}, \sqrt[\alpha^{\prime}+1]{c^{\prime} \overline{\dot{d}}_{w}^{1+\alpha^{\prime-1}}}\right\}, \\
c^{\prime}=\frac{2 \alpha^{\prime}}{\alpha^{\prime}+1} c^{-2-\alpha^{\prime-1}} \sigma_{\Xi}^{-1-\alpha^{\prime-1}} .
\end{gathered}
$$

If $\delta=\bar{\varepsilon}=\overline{\dot{d}}_{w}=0$, then a finite-time convergence of $\hat{d}_{w}$ to $d_{w}$ is substantiated.

As we can conclude, the restrictions used for the estimation algorithms are not the same. Algorithm (10) is obtained in the noise-free and constant wind conditions, despite the theoretical result this algorithm also possesses some noise filtering abilities. The estimation schemes (9), (11) assume both that the noise and wind derivative are bounded signals. Thus, some fusion of these last solutions is desirable to improve the accuracy of the wind estimates. To this end, let us define the estimates of $d_{w}$ generated by the algorithms (9), and (11), as $\hat{d}_{w}^{i}$ for $i=1,2$ respectively. Denote the errors as $v_{1}(t)=e_{a}(t)$ for (9) and $v_{2}(t)=\tilde{\Delta}_{g}(t)-\hat{\Delta}_{g}(t)$ for (11). Then

$$
\hat{d}_{w}^{\text {fusion }}(t)=\frac{\sum_{i=1}^{2} e^{-\kappa_{i} v_{i}^{2}(t)} \hat{d}_{w}^{i}(t)}{\sum_{i=1}^{2} e^{-\kappa_{i} v_{i}^{2}(t)}}
$$

is the united estimate of $d_{w}$ from all the estimation algorithms developed in this paper, and $\kappa_{i}>0$ for $i=1,2$ are tuning parameters. The proposed estimation algorithms, (9), (10) and (11), require as input an estimate of $u, v, w, \theta, \phi$, which are generally obtained fusing the accelerometric and gyroscopic measurements available on board, and ground measurements such as camera tracking.

\section{NUMERICAL EXPERIMENTS}

To show the performance of the proposed estimation algorithms, we are going to consider the Parrot Drone which has mass $m=0.47 \mathrm{~kg}$, rotors velocities $200 \mathrm{rad} / \mathrm{s} \leq \omega_{j} \leq$ $400 \mathrm{rad} / \mathrm{s}$, and we are going to compare the wind velocities in the earth frame. It means that the wind estimates $\hat{d}_{w}$ in the body frame, are then transposed to the earth frame. Gaussian noises with $2.5 \mathrm{deg} / \mathrm{s}$, and $0.052 \mathrm{~m} / \mathrm{s}^{2}$ standard deviations for gyroscope and accelerometer respectively, are added to simulate the augmented-IMU sensors noise. Additional Gaussian noises of $1 \mathrm{~cm} / \mathrm{s}$ standard deviation for linear velocities, and $1 \mathrm{deg}$ standard deviation for angles are added because state measurement $(u, v, w, \phi, \theta)$ are not reconstructed exactly. Wind signals in earth frame are simulated as sinusoids, which is a reasonable assumption 
since the gust generator at ONERA lab allows to create a sinusoidal wind gust profile, hence the sinusoidal wind effect over time is correlated to the motion of the drone through the turbulent area. Wind signals have maximal amplitude of $3,3,0.5$, since the UAV identification work by [9] is valid for $\approx \pm 5, \pm 5, \pm 1$ translational velocities respectively in $x, y, z$ axes. A flight case is illustrated where the mini drone is forced to follow the position reference in forward, lateral and vertical flights (coupling together movements on $x, y, z$ axes) in Figures 2, 3, and using PID control. Algorithms (10), (11) cannot estimate correctly the $z$ component of the wind because $w_{w}$ (wind velocity along $z$ axis in body frame) is not present in the equations of moments. Parameters of the presented algorithms are tuned by trial and errors $\left(\gamma_{a}=40\right.$, $\left.\alpha_{a}=0.9, \gamma_{g}=60, l=40, \gamma_{g}^{\prime}=40, \alpha^{\prime}=0.4, l^{\prime}=20\right)$, and kept equals for all the simulations.

Figures 4, 5 show the performance of the algorithm (9) using the three translational dynamic equations. Many varyingwind velocities, having different frequencies, are tested.

Figures 6, 7 show the performance of the algorithm (10) using the three rotational dynamic equations. The assumed noise-free and constant wind velocity conditions influence very much the quality of the result: the wind velocity with higher frequency gets the worst estimation. The tested wind velocities are the ones used for the algorithm (9).

Figures 8, 9 show that the problem of these restrictive hypotheses is solved introducing the algorithm (11), using the three rotational dynamic equations. The tested wind velocities are the ones used for the algorithm (9).

Fig. 10 show the performance of the algorithm (12), which allows to estimate correctly the wind velocity using a fusion of algorithms (9), (11) along the $x, y$ axes, and using only (9) for $z$ axis.

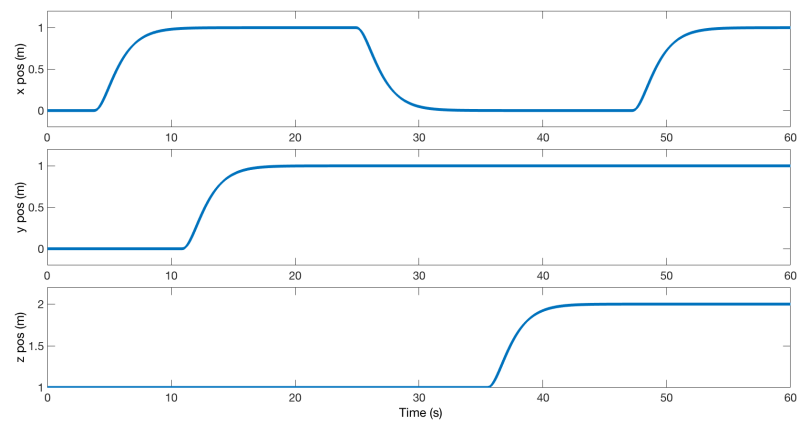

Fig. 2. Reference trajectory position in 2D.

\section{CONCLUSION}

In this work, on-line estimators of time-varying wind are designed taking the advantage of a detailed UAV aerodynamic model, hence they are strictly related to the accuracy of the UAV model representation and the identified aerodynamic coefficients. Numerical experiments are performed using nonlinear UAV simulator, modeling a quadrotor under external wind disturbance and validated through in-door experiments. The algorithms perform well the estimation of wind velocities,

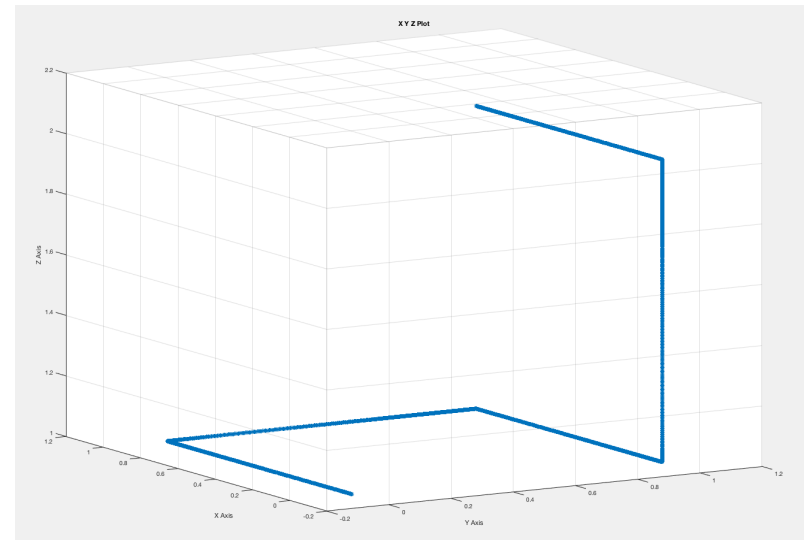

Fig. 3. Reference trajectory position in 3D.

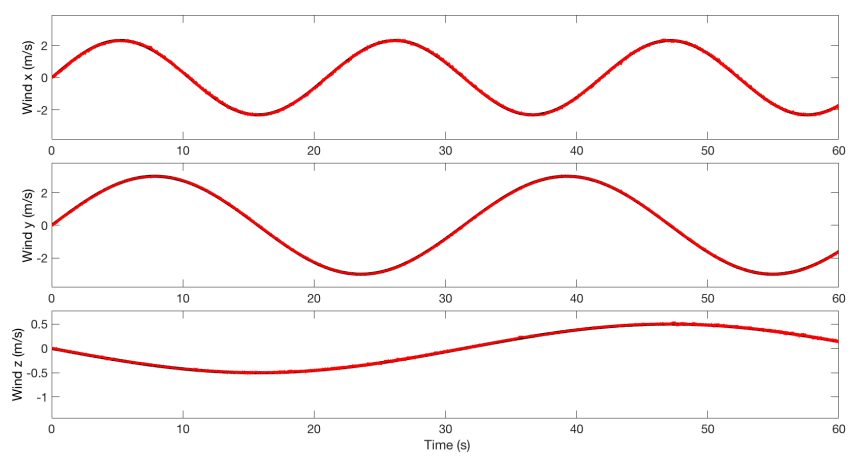

Fig. 4. Wind estimation using (9). (一: real; —: estimation).

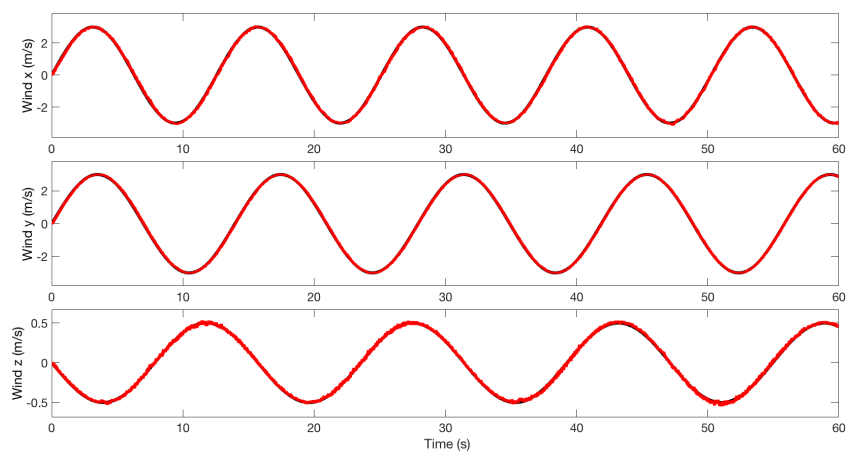

Fig. 5. Wind estimation using (9) and a reference wind velocity with higher frequency. (—: real; —: estimation).

even with the introduction of sensors' noise. Future indoor experiments will validate the proposed algorithm and further investigations can be performed by coupling this wind estimator to a robust controller ([14], [13]) to extend the domain of flight, and reducing in real-time the control effort on the rotors, when it is not necessary.

\section{REFERENCES}

[1] B. Yüksel, C. Secchi, H. H. Bülthoff, and A. Franchi, "A nonlinear force observer for quadrotors and application to physical interactive tasks," in 2014 IEEE/ASME International Conference on Advanced Intelligent Mechatronics, pp. 433-440, July 2014. DOI: 10.1109/AIM.2014.6878116. 


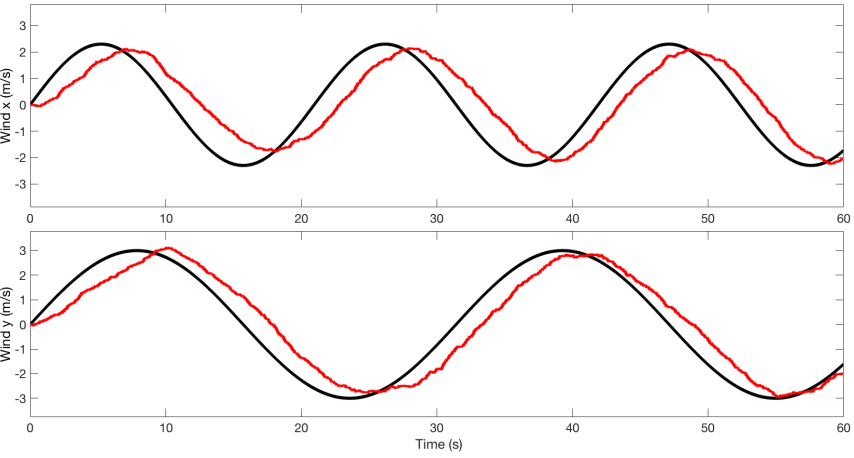

Fig. 6. Wind estimation using (10). (—: real; —: estimation).

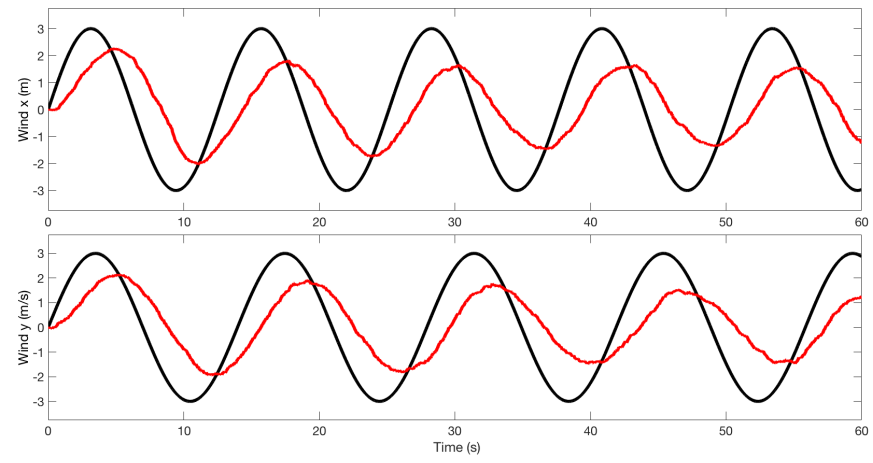

Fig. 7. Wind estimation using (10) and a reference wind velocity with higher frequency. (一: real; —: estimation).

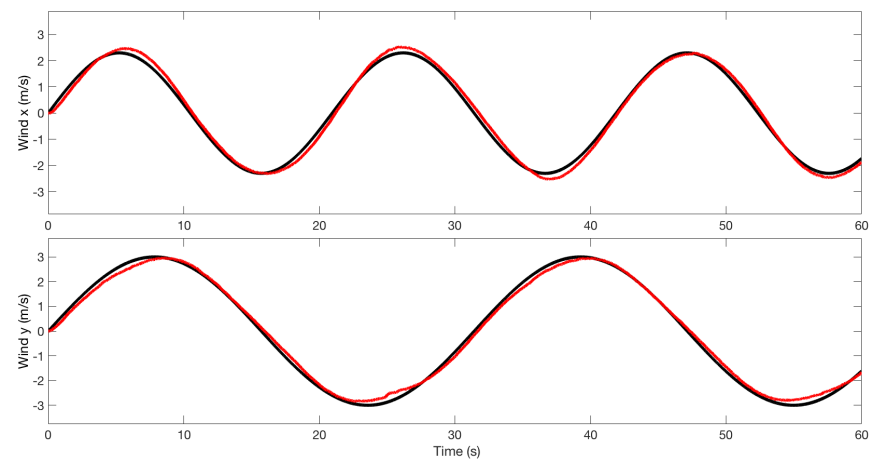

Fig. 8. Wind estimation using (11). (—: real; —: estimation).

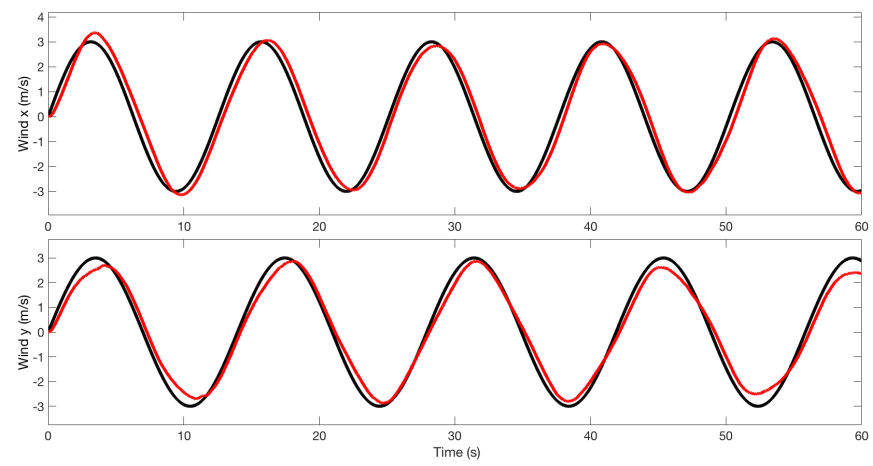

Fig. 9. Wind estimation using (11) and a reference wind velocity with higher frequency. (一: real; —: estimation).

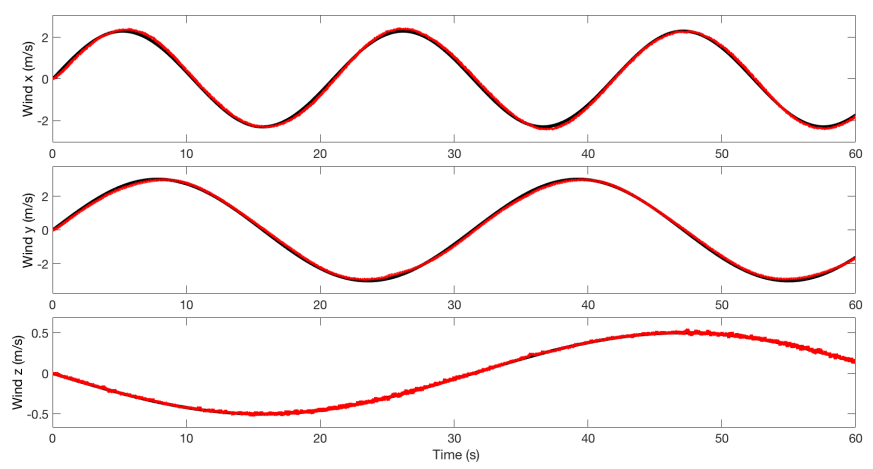

Fig. 10. Wind estimation using (12) and (9). (—: real; —: estimation).

[2] T. Tomić and S. Haddadin, "A unified framework for external wrench estimation, interaction control and collision reflexes for flying robots," in 2014 IEEE/RSJ International Conference on Intelligent Robots and Systems, pp. 4197-4204, Sept. 2014. DOI: 10.1109/IROS.2014.6943154.

[3] A. Benallegue, A. Mokhtari, and L. Fridman, "High-order slidingmode observer for a quadrotor UAV," International Journal of Robust and Nonlinear Control, vol. 18, pp. 427-440, Mar. 2008. DOI: $10.1002 /$ rnc. 1225 .

[4] H. J. Palanthandalam-Madapusi, A. Girard, and D. S. Bernstein, "Windfield reconstruction using flight data," in 2008 American Control Conference, pp. 1863-1868, June 2008. DOI: 10.1109/ACC.2008.4586763.

[5] T. A. Johansen, A. Cristofaro, K. Sørensen, J. M. Hansen, and T. I. Fossen, "On estimation of wind velocity, angle-of-attack and sideslip angle of small UAVs using standard sensors," in 2015 International Conference on Unmanned Aircraft Systems (ICUAS), pp. 510-519, June 2015. DOI:10.1109/ICUAS.2015.7152330.

[6] J. W. Langelaan, N. Alley, and J. Neidhoefer, "Wind Field Estimation for Small Unmanned Aerial Vehicles," Journal of Guidance, Control, and Dynamics, vol. 34, pp. 1016-1030, July 2011. DOI: $10.2514 / 1.52532$.

[7] S. Waslander and C. Wang, "Wind Disturbance Estimation and Rejection for Quadrotor Position Control," in AIAA Infotech@Aerospace Conference, (AIAA 2009-1983), American Institute of Aeronautics and Astronautics, Apr. 2009. DOI: 10.2514/6.2009-1983.

[8] R. Rysdyk, "Unmanned Aerial Vehicle Path Following for Target Observation in Wind," Journal of Guidance, Control, and Dynamics, vol. 29, pp. 1092-1100, Sept. 2006. DOI: 10.2514/1.19101.

[9] L. Planckaert and P. Coton, "Quadrotor UAV aerodynamic model identification using indoor flight experiment and feasibility of UAV as wind gust sensor," in International Micro Air Vehicles Conference and Flight Competition IMAV 2015, (Aachen, Germany), Sept. 2015.

[10] G. J. Leishman, Principles of Helicopter Aerodynamics with CD Extra. Cambridge University Press, Apr. 2006. ch 2-5.

[11] W. Johnson, Helicopter Theory. Courier Corporation, Mar. 2012. ch 2-5,9-13,15.

[12] A. R. S. Bramwell, D. Balmford, and G. Done, Bramwell's Helicopter Dynamics, second edition. Butterworth-Heinemann, Apr. 2001. ch 2-7.

[13] G. Perozzi, D. Efimov, J.-M. Biannic, L. Planckaert, and P. Coton, "On sliding mode control design for UAV using realistic aerodynamic coefficients," in 56th IEEE Conference on Decision and Control, (Melbourne, Australia), pp. 5403-5408, Dec. 2017.

[14] G. Perozzi, D. Efimov, J.-M. Biannic, L. Planckaert, and P. Coton, "Wind rejection via quasi-continuous sliding mode technique to control safely a mini drone," in 7th European Conference for Aeronautics and Space Science (EUCASS), (Milan, Italy), July 2017. DOI:10.13009/EUCASS2017-329.

[15] D. Efimov and A. Fradkov, "Design of impulsive adaptive observers for improvement of persistency of excitation," International Journal of Adaptive Control and Signal Processing, vol. 29, pp. 765-782, June 2015. DOI: $10.1002 /$ acs.2506.

[16] H. Ríos, D. Efimov, J. A. Moreno, and W. Perruquetti, "Finite-Time Identification Algorithm based on Time-Varying Homogeneity and Lyapunov Approach," IFAC-PapersOnLine, vol. 49, pp. 434-439, Jan. 2016. DOI: $10.1016 /$ j.ifacol.2016.10.204. 